Meta

Journal des traducteurs

Translators' Journal

\title{
À propos de mes traductions
}

\section{Valentín García Yebra}

Volume 38, numéro 4, décembre 1993

Le Je du traducteur

The $I$ of the Translator

URI : https://id.erudit.org/iderudit/004629ar

DOI : https://doi.org/10.7202/004629ar

Aller au sommaire du numéro

Éditeur(s)

Les Presses de l'Université de Montréal

ISSN

0026-0452 (imprimé)

Découvrir la revue

Citer cet article

García Yebra, V. (1993). À propos de mes traductions. Meta, 38(4), 621-629.

https://doi.org/10.7202/004629ar

Ce document est protégé par la loi sur le droit d'auteur. L'utilisation des services d'Érudit (y compris la reproduction) est assujettie à sa politique d'utilisation que vous pouvez consulter en ligne.

https://apropos.erudit.org/fr/usagers/politique-dutilisation/
Cet article est diffusé et préservé par Érudit.

Érudit est un consortium interuniversitaire sans but lucratif composé de l’Université de Montréal, l'Université Laval et l'Université du Québec à Montréal. Il a pour mission la promotion et la valorisation de la recherche. https://www.erudit.org/fr/ 


\title{
À PROPOS DE MES TRADUCTIONS
}

\author{
VALENTÍN GARCÍA YEBRA \\ Académie royale espagnole, Madrid, Espagne
}

Je peux dire que, dès mon enfance, la traduction a eu pour moi un très grand attrait. La raison en est probablement qu'aussi loin que je me souvienne j'ai ressenti un très vif intérêt pour la compréhension de ce que j'entendais ou lisais en d'autres langues, et pour ce qu'on exprimait dans la mienne.

Le village dans lequel je suis né, Lombillo de los Barrios, est situé dans la région du Bierzo où l'on parle le castillan. Cependant, c'est seulement à quelque dix kilomètres au nord-ouest que passe la frontière entre cette langue et le galicien. Lorsque j'étais enfant, très souvent des tailleurs de pierre, des scieurs, des cordiers, des rémouleurs qui parlaient galicien, venaient travailler à Lombillo. J'aimais beaucoup les entendre parler et je passais avec eux le plus de temps possible.

Il y avait chez moi quelques livres en anglais, avec de belles illustrations. C'était des livres (dont j'ai conservé certains d'entre eux) destinés à des débutants dans cette langue. Ils avaient appartenu à mon père qui avait séjourné deux ans à Cuba, désireux de là d'émigrer aux États-Unis. Lorsque mon père comprit que ce ne serait pas possible, il revint en Espagne, se maria et mourut peu de temps après, en novembre 1918. J'avais alors un an et demi. Ces livres m'attiraient tout spécialement, et aussitôt que j'ai su lire, j'ai tenté de deviner la signification de ces mots écrits à côté d'un ballon: This is a ball, à côté d'une boîte: This is a box, sous un arbre en fleurs: This is the apple tree.

À onze ans, $\mathrm{j}$ 'ai commencé mes études secondaires au collège El Espino, dirigé par les pères Rédemptoristes, dans la province de Burgos, études que $\mathrm{j}$ 'ai poursuivies à Astorga.

À El Espino, j'ai appris beaucoup de latin, moins de grec et un peu de français. Au cours de la seconde année d'enseignement secondaire, la version latine était déjà un exercice très assidûment pratiqué. Le professeur me rendait le cahier avec les deux pages de la version (qui - pour autant que je me souvienne - se faisait trois fois par semaine), décorées d'un énorme M.B. (Très Bien), tracé avec un épais crayon bleu, qui remplissait complètement les deux feuilles. Ce M.B. représentait pour moi une grande satisfaction et il m'encourageait beaucoup.

Pendant la guerre civile (j'ai été mobilisé d'avril 1937 à décembre 1939), j'ai acheté la grammaire allemande de D. Enrique Ruppert, $3^{\mathrm{e}}$ édition, Heidelberg 1909 , que j'ai toujours conservé. J'ai fini par la savoir presque par cœur. J'ai aussi conservé le cahier dans lequel $\mathrm{j}$ 'ai fait tous les exercices de traduction de l'espagnol à l'allemand. Il y en a 67 dans la première partie; 27 dans la seconde: en tout 94 . Sous le dernier figure la date du 21 juillet 1939. Ces exercices sont truffés de fautes. Malgré tout, grâce à cet effort prolongé, j'ai appris beaucoup de grammaire et de vocabulaire.

Durant l'automne de 1939, j'ai traduit pour m'exercer, mais sans penser à le publier, un roman allemand dont j'ai oublié le titre.

Entre février et mars 1940, j'ai passé et réussi l'examen d'État du baccalauréat. À cause de la date tardive, il ne m'a pas été possible de m'inscrire à l'université, mais je suis resté à Madrid toute l'année.

Pendant l'été, je n'avais rien à faire et j'étais à peu près sans argent. Il me vint à l'idée d'en gagner un peu en traduisant, pour la publier, la Médée de Sénèque. J'avais acheté à la Cuesta de Moyano ${ }^{1}$ un magnifique volume sur la couverture duquel figurait le 
titre: L. Annaei Senecae Tragoediae, Ad Optimas Editiones Collatae. Praemittitur Notitia Litteraria Studiis Societatis Bipontinae. Editio Accurata. Biponti. Ex Typographia Societatis. MDCCLXXXV. Au centre de la page titre, il y avait une gravure qui, sur fond noir, représentait Médée dans un char tiré par deux dragons ailés. Je crois que c'est cette gravure qui m'a fait traduire Médée et non une autre au sujet des tragédies qui figuraient dans le même volume. Je n'avais de préférence pour aucune d'entre elles, puisque je ne les avais pas lues.

J'ai décidé de faire cette traduction en vers. Dès l'enfance, $\mathrm{j}$ 'ai aimé versifier, mais je n'ai jamais voulu publier mes vers. Je pense qu'à deux reprises seulement j'ai failli à ce principe. La première fois, ce fut dans le volume intitulé Masa tacerii. Simposion de Metafore la Brâncusi, Bucuresti, 1970 (p. 300-302), où fut reproduit un de mes poèmes, intitulé Recuerdo a Rumanía (Souvenir de Roumanie), écrit après avoir fait deux voyages dans ce pays merveilleux et traduit en roumain par le poète Ion Caraion sous le titre: Amintirea Romániei. Dans le ${ }^{\circ} 63$ de la revue Estudios Clásicos, mai 1970 (p. 161-164), j'ai publié un autre poème, «Amarilis», inspiré du célèbre vers 5 de la première Églogue de Virgile: formosam resonare doces Amaryllida silvas.

En revanche, j'ai mis en vers, chaque fois que cela a été possible, les poèmes ou les sections de poèmes qui figuraient dans les œuvres que j'ai traduites en espagnol. Cependant traduite uniquement en vers, il n'y a que la Médée de Sénèque, et en verset claudélien, le petit livre de 104 pages de l'allemande Gertrud von Le Fort: Hymnen an die Kirche, publié en 1949 avec le $\mathrm{n}^{\circ} 56$ de la collection Adonais.

Lorsque j'ai pris la décision de traduire en vers la Médée de Sénèque, je savais peu de métrique latine. Non sans difficulté, je pouvais mesurer les hexamètres et les pentamètres. Mais d'instinct, j'ai choisi pour Médée les vers espagnols qui étaient les plus semblables au rythme présent dans ceux de Sénèque. Comme dans le texte original, il y a dans ma traduction une grande variété de mètres. Il y a beaucoup d'heptasyllabes, parfois combinés avec des hendécasyllabes, parfois seuls, comme dans le milieu du chœur du premier acte, dont le début et la fin sont en alexandrins. Le chœur du deuxième acte est écrit en dodécasyllabes, entrecoupés de temps en temps par des hexasyllabes. Les vers du chœur du troisième acte sont des hendécasyllabes saphiques, dont l'accent est sur la quatrième et la huitième syllabe, avec quelques pentasyllabes intercalés, comme dans l'original. Le chœur du quatrième acte est en hexasyllabes.

Lorsque ma traduction fut terminée, je la présentai à une maison d'édition très connue à l'époque, qui n'existe plus aujourd'hui. «Laissez-moi votre traduction et revenez dans huit ou dix jours", me dit l'éditeur. Lorsque je suis revenu, il a mis mon manuscrit sur le comptoir (il était aussi libraire) et d'un air méprisant, il me jeta ces mots : «On m'a dit que la traduction est bonne, mais cela n'a aucun intérêt du point de vue commercial.» Je suis sorti presque honteux et j'étais été sur le point de déchirer le manuscrit, dont je n'avais aucune copie. Heureusement, je ne l'ai pas fait!

Quatre ans plus tard, je suis devenu moi-même éditeur, quand trois amis et moi avons fondé, en juin 1944, les Éditions Gredos. Nul besoin de dire qu'il ne m'est pas venu à l'esprit de leur proposer d'inclure ma traduction de Médée parmi mes premiers titres. Ainsi Médée resta-t-elle inédite jusqu'en 1964, année où je devais la publier aux Éditions Gredos, à mes frais, devançant 1'hommage à Sénèque qui eut lieu en 1965, en commémoration du XIX ${ }^{\mathrm{e}}$ centenaire de sa mort. Dans le prologue de cette première édition, j'écrivais :

Il y a vingt-quatre ans, n'ayant pas encore atteint cet âge, j'ai eu la naïveté de traduire en vers cette tragédie. Je pensais qu'il ne manquerait pas d'éditeurs désireux de publier le résultat de mon travail. Lorsque, plus tard, j'ai connu le métier d'éditeur de l'intérieur, j'ai compris les causes de ma désillusion et je crois même que j'en ai ressenti un peu de honte. 
Et j'ai ajouté immédiatement:

Depuis cet été de 1940, plus de la moitié de ma vie s'est écoulée. J'ai traduit, depuis lors, de nombreuses cuvres, quelque-unes avec un assez grand succès. Cependant, je n'ai jamais travaillé avec autant d'enthousiasme qu'à la traduction de Médée. Malgré le temps, celle-ci me plaît encore beaucoup.

Le succès de cette édition n'a pas été très grand. Il a fallu dix-sept ans pour que ses trois mille exemplaires soient vendus. Malgré tout, en 1982, $\mathrm{j}$ 'en ai refait une édition très légèrement corrigée. Dans la «Note à la seconde édition», je dis :

Voici la première de mes traductions destinées à être publiées. C'est aussi ma traduction préférée. C'est pourquoi, la première édition étant épuisée depuis plus d'un an, je ne veux pas que cette cuvre soit absente du monde de la lecture [...]. Quand j'ai traduit cette tragédie, ce qui m'intéressait, ce n'était pas la critique ni l'érudition, mais la reproduction, dans des vers espagnols, de la force expressive des vers de Sénèque. Si ma passion de progéniteur ne m'aveugle pas complètement, je crois toujours que j'ai atteint mon but.

Si je me suis étendu sur le sujet de cette traduction de Médée, c'est parce que je ressens pour elle une affection toute spéciale, qui a grandi tout au long des vingt-quatre ans qu'elle a dû attendre pour être publiée.

La première traduction que j'ai faite sur commande est celle d'un grand roman de Gertrud von Le Fort, intitulé Das Schweisstuch der Veronika, publiée en espagnol sous un titre qui n'est pas le mien (El velo de Verónica). Ces modifications aux titres, effectuées pour des raisons euphoniques et commerciales, étaient courantes à l'époque. Nous autres, traducteurs, étions sans défense en face des éditeurs. J'ai traduit ce roman au printemps de 1943 et les Éditions Afrodisio Aguado l'ont publié au début de 1944. J'ai fait ce travail avec un véritable plaisir, parce que, pour la première fois, j'étais sûr qu'il allait être publié et aussi parce que le contenu du roman est très beau. J'avais l'impression de recréer l'œuvre à mesure que je la traduisais. En outre, un de ses personnages, la grand-mère de la protagoniste, femme forte et majestueuse dont la personnalité m'impressionnait beaucoup, évoquait pour moi avec une netteté extraordinaire les traits d'une grande dame d'Astorga que $\mathrm{j}$ 'admirais et que j'aimais et qui, à son tour, avait pour moi une grande affection. La grand-mère de Verónica incarnait à mes yeux doña Máxima Torbado, la mère d'un de nos grands poètes, Leopoldo Panero.

Ma traduction de El velo de Verónica a été l'objet d'éloges. Les seules personnes qui me connaissaient à Madrid étaient mes condisciples de l'université. Je devais donc penser que les éloges qu'on me faisait étaient sincères. Un critique connu, José Luis Vázquez Dodero, me félicitait si vivement que je me suis senti dans l'obligation de lui téléphoner pour le remercier. Il m'a invité un jour, mais j'étais extrêmement timide et j'ai dû faire un grand effort pour ne pas refuser son invitation. Depuis lors, nous sommes de grands amis.

La traduction de Das Jahr des Herrn (El año del Señor), du romancier autrichien Karl Heinrich Waggerl, a été aussi pour moi la source d'une très grande satisfaction. Ce texte a été publié par EPESA, en 1944, plus exactement (selon l'achevé d'imprimer) le 25 décembre. Je suppose que cette date est symbolique, puisqu'aucune imprimerie n'était ouverte le jour de Noël, ni en 1944, ni aujourd'hui. Ce livre donna lieu, plus tard, à une anecdote amusante. Nous passions l'été, ma femme et moi, avec nos deux filles aînées (mes deux autres filles n'étaient pas encore nées) dans un petit village de montagne des environs de Madrid. Il y avait là un personnage curieux, à la fois sacristain, garde-forestier et commerçant, qui me rappelait beaucoup le héros de El año del Señor. Nous étions devenus amis. Un jour le monsieur m'assure qu'il aime énormément la lecture. Je promets alors de lui prêter un exemplaire de ma traduction de Waggerl. Je le lui apporte au cours 
d'un week-end. Au moment de le lui remettre, je lui fais cette remarque: «C'est un livre excellent, mais je ne sais pas pourquoi, il ne se vend pas.» Il contemple l'ouvrage un moment de manière pensive, hoche la tête et s'exclame: «Évidemment, ce livre, je l'ai vu à la Foire du livre à Madrid et j'ai tout de suite pensé qu'il devait être intéressant. J'ai dit à la vendeuse: «Mademoiselle, je voudrais acheter ce livre.» Elle m'a répondu: «Monsieur, je suis désolée, ce livre ne se vend pas ${ }^{2} . »$

Une autre traduction de la même époque, faite dans l'enthousiasme, est Virgilio, padre de Occidente (Virgile, père de l'Occident) de l'Allemand Theodor Haecker. Elle fut publiée aussi par EPESA et (encore une fois selon l'achevé d'imprimer) le «11 novembre 1945, fête de Saint Martin de Tours». Je crois que c'est une de mes meilleures traductions. Mais le style de Haecker a une particularité. La première phrase de son prologue occupe deux lignes et demie, mais elle est suivie d'une période interminable, dans laquelle les propositions s'emmêlent en un nœud impossible à dénouer. Le lecteur se perd à coup sûr à la première lecture, et il doit alors retourner en arrière pour retrouver le fil qui lui permettra de sortir de ce labyrinthe de propositions subordonnées, de subordonnées de subordonnées, et de subordonnées de subordonnées de subordonnées. Cette période du prologue s'étend sur quarante-quatre lignes. Il y en a d'autres moins longues, un peu moins emmêlées, mais qui ne sont jamais simples. Il suffit de dire que le prologue occupe dix pages et demie et n'a que trois points finals. Cette manière d'écrire reste la même tout au long du livre, mais jamais elle n'est aussi caractéristique que dans le prologue. Il semble que l'auteur veuille nous obliger à parcourir un chemin interminable avant de nous laisser admirer les merveilleux trésors que renferme ce petit mais splendide palais qu'est Virgilio, padre de Occidente.

La critique a fait de grands éloges de ma traduction, mais elle m'a reproché d'avoir suivi de trop près la syntaxe du texte allemand. À l'époque, je n'avais pas encore de théorie établie sur le sujet, et quand, trois ans plus tard, j'ai traduit un autre livre de Haecker, $L a$ joroba de Kierkegaard (La bosse de Kierkegaard), j'ai tenu compte de ces critiques et démembré les longues périodes de l'auteur. Aujourd'hui, je pense que le procédé peut être légitime dans un livre de contes, et même dans certains romans, dont la finalité est de plaire au lecteur, mais qu'il ne l'est pas quand on traduit un auteur dense et profond comme Haecker. Le traducteur ne doit pas fausser le style d'un auteur. Le lecteur qui recule devant la sévérité en apparence extérieure de la syntaxe de Haecker serait, de toute manière, incapable de mesurer la profondeur de sa pensée et même d'apprécier les subtils éclats de lumière qui en irisent fréquemment la surface.

Il m'est impossible de commenter chacune de mes traductions de l'allemand. C'est la langue à partir de laquelle j'ai le plus souvent travaillé. Je mentionnerai cependant un excellent essai de Gertrud von Le Fort, intitulé Die ewige Frau ( «La mujer eterna», La femme éternelle), dont on n'a imprimé - je ne sais par quel étrange caprice de l'éditeur — quie cent exemplaires numérotés. C'est une édition faite à Madrid en 1947.

J'ai également traduit des œuvres philosophiques, comme celle de G. M. Manser: $L a$ esencia del tomismo (L'essence du thomisme) (Madrid, 1946), qui a connu immédiatement une réimpression et celle de $\mathrm{H}$. Rommen, La teoría del Estado y de la Comunidad Internacional en Francisco Suárez (La théorie de l'État et de la communauté internationale chez Francisco Suárez) (Madrid, 1951). En ce qui concerne ma spécialité officielle, je suis le traducteur de W. Brandenstein: Lingüistica griega (Linguistique grecque) (Madrid, 1965).

Je n'ai traduit que peu de choses de l'anglais: un essai signé D. A. Callus et intitulé La condena de Santo Tomás en Oxford (La condamnation de Saint Thomas à Oxford), lequel fut publié dans le $\mathrm{n}^{\circ} 22$ de la Revista de Filosofía (p. 379-416), et des articles qui ont paru dans la revue Arbor, entre 1948 et 1955, années durant lesquelles j'en étais le rédacteur. Sauf en de rares exceptions, ces travaux ne portent pas ma signature. Je ne me 
souviens pas des titres, pas plus que des auteurs traduits. J'ai seulement les tirés-à-part d'un article intitulé «Valera en Washington» (Valera à Washington) de Cyrus DeCoster (Arbor n ${ }^{\circ}$ 58, p. 215-224).

En revanche, j'ai traduit un nombre assez grand d'œuvres françaises - pas autant, cependant, que d'œuvres allemandes. Mis à part les nombreux articles pour Arbor, je suis auteur d'une traduction de E. Gilson: El realismo metódico (Le réalisme méthodique) (Madrid, 1950) et d'une autre de L. de Raeymaeker: Filosofía del ser (La philosophie de l'être) (Madrid, 1954), réalisée en collaboration avec ma femme, María Dolores Mouton.

La traduction la plus importante du français fut, pour moi, en raison de ses répercussions, celle de l'ensemble des cinq tomes de l'œuvre monumentale du prêtre belge Charles Moeller: Littérature $d u X X^{e}$ siècle et christianisme. Lorsque j'ai pris la décision de traduire et de publier cette œuvre aux Éditions Gredos, en 1955, deux tomes de l'édition originale avaient déjà paru. Pour gagner du temps, j'ai confié à mon ami José Pérez Riesco la traduction du deuxième tome et me suis réservé le premier. Pérez Riesco a fait une excellente traduction. Mais au fil des éditions suivantes, Moeller augmenta considérablement le tome II qui, dans la première édition, était beaucoup plus réduit que le tome I, alors que, dans la sixième, il atteint presque 500 pages. Je suis le traducteur de tout ce qui fut ajouté après la première édition.

Dans les cinq tomes publiés (l'auteur en avait prévu un dernier qui devait clôre l'ensemble 3 ), Moeller consacre des essais aussi profonds qu'humains et profondément respectueux, à une longue série d'écrivains contemporains. Il analyse et interprète leurs œuvres dans une perspective essentiellement chrétienne, catholique, mais aussi de tolérance et de compréhension. Peu après la publication en français des deux premiers tomes de l'œuvre de Moeller, le bruit a couru qu'ils avaient été sur le point d'être mis à l'Index, qui prévalait encore. Je ne sais ce qu'il y a de vrai dans cette rumeur. Il est curieux, en tout cas, que quelques années plus tard Moeller ait occupé au Vatican la fonction - très importante - de sous-secrétaire de la Congrégation pour la Doctrine de la Foi et que cet Index fameux ait été rapidement aboli.

Il n'est pas sans intérêt non plus de rappeler les auteurs étudiés par Moeller dans chaque tome :

- dans le premier: Albert Camus, André Gide, Aldous Huxley, Simone Weil, Graham Greene, Julien Green et Georges Bernanos;

- dans le deuxième: Jean-Paul Sartre, Henry James, Roger Martin du Gard et Joseph Malègue;

- dans le troisième: André Malraux, Franz Kafka, Vercors, M. Sholokhov, Thierry Maulnier, Alain Bombard, Françoise Sagan et Stadislas Reymont;

- dans le quatrième: Anne Frank, Miguel de Unamuno, Gabriel Marcel, Charles du Bos, Fritz Hochwälder et Charles Péguy;

- dans le cinquième, de nouveau Françoise Sagan, Bertolt Brecht, Saint-Exupéry, Simone de Beauvoir, Paul Valéry et Saint-John Perse.

Un total de trente auteurs de première importance sont étudiés en 2700 pages sereines et bien structurées. L'auteur se proposait de consacrer un sixième volume à Marguerite Duras, Ignazio Silone, T. S. Eliot, Sigrid Undset et Gertrud von Le Fort.

On a rarement vu une telle unanimité dans les éloges de critiques espagnols et latinoaméricains de toutes tendances. Les Éditions Gredos ont imprimé, après la publication en espagnol du quatrième volume, une Antología de juicios críticos sobre «Literatura del siglo XX y cristianismo» (Anthologie de critiques sur la «Literatura del siglo XX y cristianismo») qui occupe quatorze pages en petits caractères. Le texte de la courte «note d'introduction» est le suivant: 
Pour des raisons d'espace, nous avons dû omettre de nombreuses critiques, aussi enthousiastes que celles qui figurent ici. Il suffit de dire que, uniquement pour le tome IV, nous avons, dans nos fichiers, plus de deux cents comptes rendus publiés en Espagne, sans compter ceux publiés en Amérique latine. À l'exception de deux ou trois d'entre eux, dont le ton est celui qu'on attendait, tous sont unanimes dans l'éloge enthousiaste.

Il serait impossible de reproduire des extraits de ces critiques. Cependant je ne peux résister à la tentation de transcrire ce qu'écrivit, en juillet 1955, José Luis Martín Descalzo, tout récemment disparu. Ce critique, alors très jeune, déclarait dans la revue Incunable (vol. II, $\mathrm{n}^{\circ} 76$ ) :

Je crois que je n'ai jamais fait la critique d'aucun livre avec autant d'émotion, autant de sentiment que celui que j'éprouve en prenant la plume pour parler du livre de Ch. Moeller. C'est un livre réellement, véritablement, hors série.

En février 1961, Charles Moeller vint à Madrid et prononça deux conférences à la Faculté de Philosophie et Lettres de 1'Université Complutense. Il y eut une émeute dans la salle, les étudiants se disputant l'espace pour pouvoir écouter l'abbé belge. Beaucoup entrèrent par la fenêtre. Le journal Ya écrivait le 10 mars dans son éditorial :

Charles Moeller est à Madrid pour donner quelques conférences à l'université. L'Abbé Moeller est l'auteur de Literatura del siglo XX y Cristianismo. Inutile d'en dire plus. Ce livre, encore sous presse, est probablement l'œuvre catholique de ces dernières années qui a eu la plus profonde influence sur l'intelligence et la sensibilité des jeunes universitaires qui, en tenant la main du professeur belge, ont pu se frayer un chemin dans la jungle du roman et de l'essai contemporains.

Quelques jours plus tard, le 27 février, le poète José García Nieto, devenu aujourd'hui académicien, écrivait dans El Alcázar:

Amphithéâtre de la Faculté débordant de jeunes gens tous prêts à écouter la conférence du penseur et critique belge Ch. Moeller, connu en Espagne grâce à Literatura del siglo XX y cristianismo. Ch. Moeller est l'une des figures les plus cultivées et les plus nécessaires du monde intellectuel de notre époque. Personne mieux que lui - croyons-nous - n'a-t-il abordé le sujet de la littérature actuelle. Il l'a fait avec une finesse, une profondeur et une générosité peu communes.

Quant aux éloges décernés à ma traduction, je rougirais de les reproduire. Pour la traduction des quatre premiers tomes de cette cuvre (le cinquième ne sera publié en français qu'en 1975), j'ai obtenu, en mars 1964, le Prix annuel de la traduction décerné par le Ministère belge de l'éducation et de la culture pour «attirer l'attention sur les meilleures traductions d'œuvres belges ${ }^{4}$ ». Dans la lettre m'annonçant que le prix m'avait été attribué, on écrivait: "Vous êtes le premier à bénéficier de cette haute distinction ${ }^{4} . »$ Comme il est fréquent en Espagne que les prix soient donnés par amitié ou sous recommandation, on me demanda alors si j'avais des amis en Belgique. Je répondis que la reine Fabiola avait créé ce prix à mon intention. Quelques-uns crurent qu'il en était ainsi. Le prix représentait une somme importante pour l'époque: presque cent mille pesetas. J'ai consacré un peu plus du tiers de cet argent à la première édition de Médée.

Je n'ai pas parlé jusqu'à maintenant d'une traduction que l'on pourrait dire «mixte» parce qu'elle n'est pas entièrement de moi et qu'elle n'est pas faite non plus à partir d'une même langue. Il s'agit de Interpretación y análisis de la obra literaria de Wolfang Kayser dont la première édition fut faite à Madrid (Gredos, 1950) et la quatrième, en 1967 , avec de nombreuses réimpressions pour chaque édition. Cette traduction fut faite en collaboration avec ma femme, en partie de l'allemand et en partie du portugais. L'auteur 
était professeur à l'Université de Lisbonne au moment de la parution de la première édition en portugais. Plus tard Kayser a refondu l'ouvrage dans sa version allemande. Nous avons comparé les deux éditions, la portugaise et l'allemande, et avons choisi ce qui nous paraissait le meilleur dans chaque cas, complétant parfois la théorie et remplaçant les exemples empruntés à la littérature étrangère par d'autres puisés dans la littérature espagnole. Ce livre de Kayser est un classique de la théorie littéraire qui a encore un grand succès aujourd'hui, de nombreuses années après la mort de son auteur.

En ce qui concerne les contenus et le succès, le livre de Kayser a certains points en commun avec Teoría de la literatura, de V. M. de Aguiar e Silva, professeur à l'Université de Coímbre. Ma traduction espagnole de ce livre a été publiée chez Gredos, en 1972. Elle a connu depuis sept réimpressions. Une autre caractéristique commune aux deux œuvres est la présence fréquente de poèmes en allemand, en anglais, en français, en italien, en portugais, reproduits à titre d'exemples. Je les ai presque toujours traduits en vers. $\mathrm{Si}$ on les réunissait, ils constitueraient un volume de dimension considérable.

Bien que cela nous fasse faire un saut dans le temps, je mentionnerai, en dernier lieu, parmi mes traductions en langues romanes, celle du livre de mon ami Oronzo Giordano, Religiosità popolare nell' Alto Medioevo, traduit en collaboration avec ma fille, Pilar García Mouton (Madrid, Gredos, 1983, 312 pages).

Mon séjour de onze ans à Tanger, de 1955 à 1966, comme directeur de l'Institut Polytechnique Espagnol, fécond sous d'autres aspects, a cependant réduit mon activité de traducteur. Durant cette période je n'ai traduit que les ajouts au volume II et les volumes III et IV de Moeller. J'ai également fait une étude approfondie de la Métaphysique d'Aristote et de ses traductions latines, qui me servit non seulement lors de ma soutenance de thèse, présentée en 1967, mais aussi pour l'édition trilingue de l'œuvre du grand philosophe que j'ai publiée en 1971. Pour cette traduction, j'ai obtenu le prix Ibáñez Martín du CSIC5. Ce prix avait été institué l'année auparavant, et, comme pour le Prix annuel de la Traduction belge, c'est à moi qu'il fut décerné pour la première fois. La Métaphysique en version trilingue a connu une deuxième impression en 1982 et une troisième en 1987.

J'ai publié également une édition trilingue de la Poétique d'Aristote. Métaphysique et Poétique sont les deux pôles - l'un philosophique et l'autre littéraire - du monde intellectuel d'Aristote. Dans ces deux éditions trilingues, il y a plus de travail que dans une simple traduction espagnole. Pour cette raison, j'ai l'habitude de les compter parmi mes œuvres de création et non parmi mes traductions, mais elles appartiennent cependant à ces deux domaines, principalement parce que ce sont mes seules traductions du grec, langue de ma spécialité officielle.

Traduire des textes dans des langues classiques, spécialement en grec, est en soi beaucoup plus difficile qu'à partir de langues romanes et même germaniques.

En premier lieu, le traducteur moderne, en particulier de langue romane, achoppe sur la distance typologique instaurée entre sa langue et le grec. Les langues romanes sont plus analytiques, elles ont perdu leur déclinaison nominale et expriment les relations entre les éléments de la phrase au moyen des prépositions. Le grec classique, plus analytique que le latin (il utilisait davantage les prépositions et surtout disposait de ce précieux instrument qu'est l'article), était, cependant, une langue fondamentalement synthétique. La déclinaison nominale, même si elle comptait un cas de moins qu'en latin, était encore vivante en grec, langue qui présente en outre la difficulté d'un lexique beaucoup plus éloigné du nôtre que ne l'est le lexique latin.

La traduction d'Aristote est particulièrement difficile à cause des sujets traités et du style propre à de ce philosophe. Menéndez Pelayo écrivait, en 1875, à propos de la Métaphysique, qu'elle n'avait jamais été traduite en espagnol «peut-être parce que son obscurité et son style aride ont découragé nos humanistes». La difficulté de la Poétique est sans 
aucun doute moindre, mais elle est néanmoins aussi réelle. Les deux appartiennent au groupe d'œuvres que les premiers commentateurs d'Aristote ont appelées «ésotériques» ou «acroamatiques». Elles présentent, en outre, un caractère fragmentaire et incomplet, ce qui est, pour le traducteur, source de nombreuses difficultés.

Heureusement, ces difficultés sont compensées par l'existence d'instruments dont le traducteur de textes modernes est dépourvu. En premier lieu, les grandes ceuvres des auteurs classiques - et c'est le cas d'Aristote - ont été en général étudiées par les grands commentateurs. La consultation de ces derniers est extrêmement utile. Les traducteurs de grec disposent aussi de bons dictionnaires généraux et spécialisés. Il est très difficile de parvenir à une telle maîtrise du grec ancien que l'on puisse se passer complètement d'un bon dictionnaire. Pour traduire Aristote, aucun dictionnaire n'est comparable à l'Index Aristotelicus de Hermann Bonitz, republié en 1961 par les Éditions Walter de Gruyter (Berlin). Les différents usages de chacun des mots qui figurent dans l'œuvre d'Aristote s'y trouvent repertoriés. L'importance du contexte dans la théorie et dans la pratique de la traduction est bien connue. La signification d'un mot prend une nuance particulière, elle se transforme sous l'influence des mots qui l'entourent, c'est-à-dire du microcontexte. Pour connaître l'effet du microcontexte, la perspicacité d'un traducteur soigneux suffit en général. Cependant, souvent dans Aristote, il se présente des termes d'interprétation douteuse, pour l'élucidation desquels il ne suffit pas de tenir compte du microcontexte. Il faut alors recourir au macrocontexte, c'est-à-dire à la situation du terme difficile dans l'ensemble de l'œuvre traduite et parfois dans l'ensemble des différentes œuvres de l'auteur. Dans ce cas, l'Index Aristotelicus est un instrument précieux au plus haut point. Le traducteur peut y trouver immédiatement tous les passages d'Aristote dans lesquels apparaît le terme concerné.

Un autre instrument, qui n'est pas à dédaigner non plus, est l'ensemble des traductions antérieures de la même œuvre. Je dois avouer que, lorsque je traduisais la Métaphysique et la Poétique, j'ai consulté, pour résoudre les passages difficiles, toutes les traductions possibles: latines, allemandes, anglaises, italiennes, françaises. Aucun traducteur ne doit se considérer infaillible et, lorsqu'il interprète des œuvres comme celle d'Aristote, il ne doit dédaigner aucune aide. En ce qui concerne les traductions déjà faites dans la même langue, le traducteur doit les connaître toutes. En premier lieu, parce que s'il en existe une qui fait autorité, il n'y a aucune raison d'en entreprendre une nouvelle, et il vaut mieux alors que le traducteur concentre ses efforts sur un autre sujet.

Les traductions les plus utiles à la compréhension d'Aristote sont, en général, les traductions latines. Aucune langue moderne, et les langues romanes moins que les autres, ne peuvent suivre le texte grec de si près et avec autant d'exactitude que le latin, mais il est évident que, si l'on ne connaît pas bien le grec, aucune traduction ne sera suffisante.

Lorsqu'on consulte les traductions antérieures, il est nécessaire d'être prévenu d'un danger possible. Le nombre ou l'autorité des prédécesseurs peut parfois éloigner le traducteur de l'interprétation correcte. Dans mon édition trilingue de la Poétique (p. 316, note 289), je signale les doutes que j'ai eus avant de m'éloigner de l'interprétation traditionnelle de l'adjectif verbal syntheté. Pour les traducteurs latins, français, allemands, anglais, italiens, etc., le terme aristotélicien phoné syntheté équivalait à «mot composé»: vox composita (Riccoboni), quae componi potest (Heinsius), un composé de sons (Hardy), composite sound (Else), zusammengesetzter Laut (Gigon), composto (Sousa), voce composita (Pittau). Le premier traducteur espagnol de la Poétique, Alonso Ordóñez das Seijas y Tobar, avait, étonnament, omis la traduction de syntheté; mais Casimiro Flórez Canseco, reprenant un siècle et demi plus tard - en 1778 - la traduction d'Ordóñez, avait écrit sans hésiter: «Voz compuesta» (mot composé). Il avait insisté dans une note: «Le grec dit: mot composé, syntheté.» Vingt ans plus tard, en 1798, Goya y Muniain donnait exactement la même traduction. 
Personnellement, je pense qu'Aristote, en définissant le nom, ne voulait pas dire qu'il s'agissait d'un «mot composé» mais d'un «mot conventionnel». Ainsi avait-il, plus de deux mille ans avant Saussure, conçu l'idée de l'arbitraire du signe qu'il formula cependant avec plus de justesse, les signes linguistiques n'étant pour lui pas «arbitraires», mais «conventionnels». Je ne peux reprendre ici les arguments sur lesquels s'appuie mon interprétation. Mais je voudrais insister sur le fait que le traducteur qui consulte ceux qui l'ont précédé doit conserver son esprit critique malgré l'autorité et le nombre de ses prédécesseurs. De la même manière, il est important de ne pas se borner à prendre en considération le microcontexte; il faut également tenir compte du macrocontexte, lequel peut parfois être constitué non seulement par l'œuvre que l'on traduit, mais encore par d'autres œuvres du même auteur.

Peut-être certains lecteurs s'attendent-ils à ce que je leur explique comment, selon moi, il faut traduire. C'est une chose qui ne peut s'expliquer en quelques lignes. Avec le souci d'enseigner la traduction, l'Université Complutense de Madrid créa, en mai 1974, 1'Institut Universitaire de Langues Modernes et de Traduction, où j'ai fait part, dès le mois d'octobre de la même année, de ma «théorie de la traduction», que j'ai constamment tenté d'associer à la pratique. Certaines de mes idées et le contenu de quelques-uns de mes cours se retrouvent dans les quasi neuf cents pages que constituent les deux tomes de mon livre, Teoría y práctica de la traducción ${ }^{6}$, dans le tome complémentaire, En torno a la traducción ${ }^{7}$, qui ajoute près de quatre cents pages aux ouvrages précédents, et, enfin, dans une douzaine d'articles qu'il reste à réunir. Et je n'ai pas encore tout dit...

Pour la première des œuvres que je viens de mentionner et, qui a connu une seconde réimpression deux ans après, l'Académie Espagnole me décerna le Prix «Lorenzo Nieto López», «destiné - s'il faut en croire l'annuaire de l'Académie - à la personne, naturelle ou juridique qui, par ses travaux en faveur de la langue espagnole, s'est rendue, aux yeux de l'Académie, digne de cette distinction».

Finalement, j'ajoute que mon discours d'entrée à l'Académie Espagnole traite de la traduction comme facteur d'enrichissement de la langue du traducteur.

Il y a cependant une formule que j'ai répétée en de multiples occasions et qui résume ma pensée sur le «comment traduire». La voici :

Le traducteur doit dire tout ce que dit le texte original, il doit ne rien dire que l'original n'ait dit et doit tout dire de la manière la plus naturelle et, quand l'original le permet, de la manière la plus élégante possible dans la langue dans laquelle il traduit.

(Traduction de Brigitte Lépinette)

\section{Notes}

I. N.d.T. Lieu à Madrid où l'on vendait (et où l'on vend encore) des livres d'occasion.

2. N.d.T. No se vende est polysémique en espagnol et peut signifier: 1) qu'il ne se vend pas bien (il n'a pas de succès), ou 2) qu'il n'est pas en vente. Évidemment le traducteur interprète la phrase dans le premier sens, alors que le sacristain pense que la vendeuse a employé la phrase dans le second sens.

3. Moeller est mort en 1986 en laissant son cuvre inachevée.

4. En français dans le texte.

5. Le C.S.I.C. (Consejo superior de Investigaciones Cientificas) est l'équivalent du CNRS français.

6. Théorie et pratique de la traduction.

7. A propos de la traduction. 